\title{
Hemorragia gastrointestinal como primera manifestación de coriocarcinoma metastásico en un paciente masculino
}

\author{
Gastrointestinal hemorrhage as the first manifestation of metastatic choriocarcinoma in a \\ male patient
}

\author{
Uriel Reyes-Ramírez" ${ }^{*}$, Hugo Enrique Estrada-González', José Cruz-Santiago, \\ José Rafael Peñavera-Hernández ${ }^{2}$, Gabriela Gutiérrez-Muñiz ${ }^{2}$ y Víctor Manuel Pinto-Angulo ${ }^{1}$ \\ ${ }^{1}$ Servicio de Cirugía General; ' 2 Servicio de Anatomía Patológica. Hospital Juárez de México, Ciudad de México, México
}

\begin{abstract}
Resumen
Introducción: El coriocarcinoma es un tumor maligno, más frecuente en el sexo femenino, raramente reportado en el sexo masculino. Caso clínico: Varón de 19 años que ingresa con hematoquecia y evacuaciones melénicas. Se realiza panendoscopia y colonoscopia, sin documentar el sitio de sangrado. Se realiza laparotomía exploradora y se encuentra una lesión tumoral en el yeyuno, cuyo examen histopatológico reportó coriocarcinoma. Conclusiones: La hemorragia de tubo digestivo como presentación de un coriocarcinoma es en ocasiones el único síntoma que muestra un paciente. El coriocarcinoma metastásico al tracto gastrointestinal es raro, lo que hace que la sospecha diagnóstica sea pobre.
\end{abstract}

Palabras clave: Hemorragia gastrointestinal. Coriocarcinoma. Masculino. Intestino delgado. Metástasis.

\begin{abstract}
Introduction: Choriocarcinoma is a malignant tumor, it is more frequent in the female sex, rarely reported in the male sex. Clinical case: A 19-year-old male patient who was admitted with hematochezia and melenic evacuations, panendoscopy and colonoscopy were performed without documenting the bleeding site, exploratory laparotomy was performed finding tumor lesion in the jejunum, the histopathological examination reported Choriocarcinoma. Conclusions: Gastrointestinal bleeding as a presentation of choriocarcinoma is sometimes the only symptom that the patient presents. Metastatic choriocarcinoma to the gastrointestinal tract is rare, which makes the suspected diagnosis poor.
\end{abstract}

Key words: Gastrointestinal bleeding. Choriocarcinoma. Male. Small intestine. Metastasis.

\section{Introducción}

El coriocarcinoma es un tumor maligno de células trofoblásticas que es más frecuente en el sexo femenino, principalmente en embarazos molares o embarazos ectópicos, raramente reportado en el sexo masculino, en el cual su forma más típica de presentación es en el testículo. El 93\% de los tumores testiculares son de células germinales, pero el coriocarcinoma solo representa el $1 \%$ de los casos de tumores de células

\section{Correspondencia:}

*Uriel Reyes-Ramírez

Avda. Instituto Politécnico Nacional, 4829

Col. Tlacamaca Gustavo A. Madero

C.P. 07380 , Ciudad de México, México

E-mail: ur1rr@ hotmail.com
Fecha de recepción: 18-07-2020

Cir Cir. 2021;89(S1):10-13

Fecha de aceptación: 18-09-2020

DOI: 10.24875/CIRU.20000786
Contents available at PubMed www.cirugiaycirujanos.com

0009-7411/@ 2020 Academia Mexicana de Cirugía. Publicado por Permanyer. Este es un artículo open access bajo la licencia CC BY-NC-ND (http://creativecommons.org/licenses/by-nc-nd/4.0/). 
germinales, con una incidencia de 2.1 casos por cada 100,000 hombres, siendo cuatro veces más frecuente en los hombres blancos que en los de raza negra. Las presentaciones extragenitales primarias mayormente descritas son el trayecto de la línea media en el retroperitoneo, el mediastino, la glándula pineal y el tracto gastrointestinal; de este último, el estómago es el órgano afectado mayormente descrito. No obstante, existen pocos casos reportados, sobre todo en la literatura inglesa, y de todos los casos, solo el $5 \%$ desarrollan manifestaciones gastrointestinales. La presentación clínica suele ser tardía y debutar con sangrado del tubo digestivo de origen incierto. El pronóstico es pobre ${ }^{1-5}$.

En su forma testicular, la edad de presentación oscila entre los 15 y los 35 años, y tiene una supervivencia a 5 años menor del $80 \%$, según la epidemiología norteamericana, por el diagnóstico tardío. Su evolución suele ser rápida y con progresión metastásica acelerada, sobre todo en tumores no seminomatosos de células germinales. Existe elevación de la $\alpha$-fetoproteína $>10,000 \mathrm{ng} / \mathrm{ml}$, la fracción $\beta$ de la gonadotropina coriónica humana $>50,000 \mathrm{IU} / \mathrm{l}$ y la deshidrogenasa láctica $(\mathrm{DHL})>10$ veces arriba de su valor de referencia. En los pacientes con afección tumoral extendida y hemorragia gastrointestinal, el abordaje endoscópico debe ser individualizado para identificar el sitio de sangrado, tomar biopsias y realizar terapia endoscópica de ser necesaria, aunque el diagnóstico histológico en la mayoría de los casos casualmente se consigue post mortem por reportes de los patólogos ${ }^{6-9}$.

Existen casos de coriocarcinoma de tracto gastrointestinal que ocurren en asociación con un carcinoma o como presentación aislada. Además de en el estómago, también se han reportado tumores en el esófago, el yeyuno y el colon. Presentamos el caso de un paciente varón de 19 años con coriocarcinoma de yeyuno de evolución rápida que debutó con hemorragia gastrointestinal, sometido a laparotomía exploradora y resección en el Hospital Juárez de México, con reporte histopatológico por el servicio de patología de la unidad médica ${ }^{10}$. Se trata de la exposición de un caso clínico poco común, con hemorragia de tubo digestivo alto como primera manifestación de coriocarcinoma metastásico en paciente de sexo masculino.

\section{Caso clínico}

Varón de 19 años, sin antecedentes crónico-degenerativos, que acude al servicio de urgencias por haber presentado un episodio de hemorragia de tubo digestivo alto, manifestado por hematoquecia y melena no cuantificada de 2 días de evolución, así como astenia, adinamia e hiporexia. En la exploración física no se observan alteraciones neurológicas ni respiratorias; la auscultación cardiaca muestra ruidos aumentados en intensidad y frecuencia de 110 latidos por minuto. La presión arterial es de 130/75 mmHg. La exploración abdominal muestra un abdomen plano, blando, depresible, no doloroso a la palpación, con hepatomegalia palpable a $2 \mathrm{~cm}$ del reborde costal, peristalsis presente, sin masas palpables. El testículo derecho presenta un aumento de volumen con respecto al lado contrario, no doloroso, sin cambios de coloración en la piel. No hay evidencia de adenomegalias. El tacto rectal revela presencia de melena, sin otra alteración. Los datos de laboratorio fueron: hemoglobina $7.1 \mathrm{~g} / \mathrm{dl}$, hematocrito $21.6 \%$, volumen corpuscular medio $89.5 \mathrm{fL}$, hemoglobina corpuscular media $29.4 \mathrm{pg}$, leucocitos 12,500, plaquetas 227,000 , tiempo de protrombina $27.5 \mathrm{~s}$, international normalized ratio 2.61 , tiempo de tromboplastina parcial $42.8 \mathrm{~s}$, electrolitos normales, creatinina $0.9 \mathrm{mg} / \mathrm{dl}$, glucosa $101 \mathrm{mg} / \mathrm{dl}$, nitrógeno ureico en sangre $36 \mathrm{mg} / \mathrm{dl}$, urea $75 \mathrm{mg} / \mathrm{dl}$ y DHL 2355 UI/l. Posterior a la reanimación con líquidos y transfusión de sangre, se realizó una endoscopia digestiva alta, la cual reveló una lesión subepitelial en la curvatura menor del estómago sin evidencia de sangrado. Se decidió realizar una colonoscopia, que mostró abundantes coágulos en la flexura cólica izquierda que dificultaban el paso del colonoscopio (Fig. 1). El paciente se ingresó a cargo del servicio de medicina interna y continuó con sangrado del tubo digestivo alto y choque hipovolémico, por lo que se interconsultó al servicio de cirugía general y este decidió realizar una laparotomía exploradora, en la que se encontró un tumor de aproximadamente $4 \times 5 \mathrm{~cm}$ a $60 \mathrm{~cm}$ del ángulo de Treitz. Se realizó una resección intestinal de aproximadamente $8 \mathrm{~cm}$, que incluyó el tumor, y posteriormente entero-enteroanastomosis termino-terminal. Continuando con la revisión de la cavidad abdominal, se evidenciaron metástasis hepáticas (Fig. 2). Se envió el tejido resecado al servicio de anatomía patológica (Fig. 3). La evolución del paciente fue desfavorable y falleció al día siguiente. El examen histopatológico reportó coriocarcinoma de $4.5 \mathrm{~cm}$ de eje mayor que infiltraba hasta la serosa peritoneal, con infiltración linfovascular.

\section{Discusión}

Los tumores de células germinales no son una afección maligna rara, pero el desarrollo de un coriocarcinoma en el sexo masculino sí lo es, como describen 


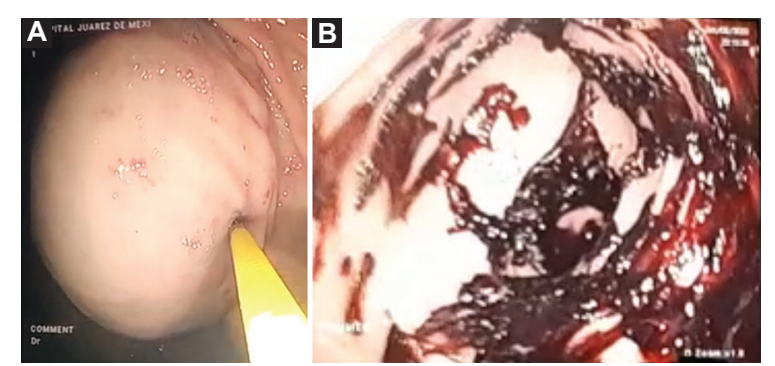

Figura 1. A: panendoscopia que reporta una lesión subepitelial de $60 \mathrm{~mm}$ en la curvatura menor del estómago, infracardial. B: abundantes coágulos en la flexura cólica izquierda que impiden el paso del colonoscopio.
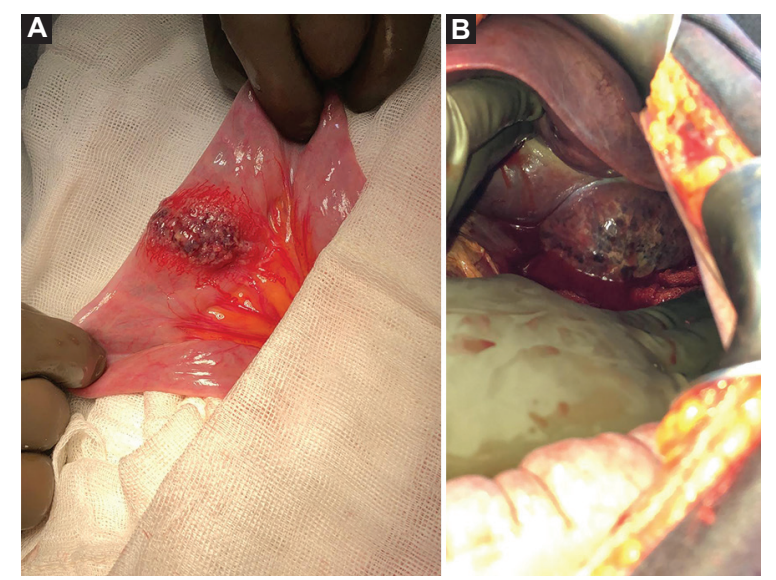

Figura 2. A: imagen transoperatoria de la lesión en el yeyuno, de aproximadamente $4 \times 5 \mathrm{~cm}$. B: metástasis en el lóbulo izquierdo del hígado.

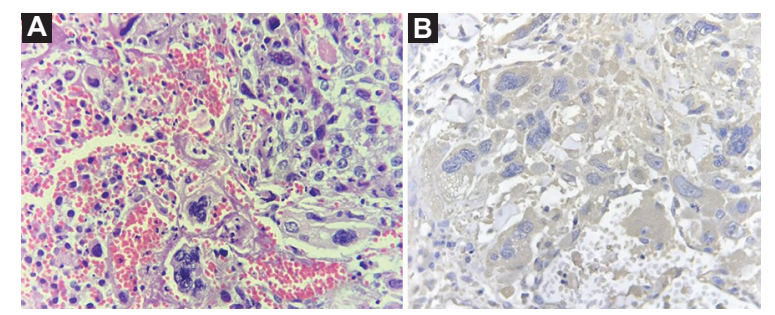

Figura 3. A: imagen teñida con hematoxilina-eosina que muestra células del sincitiotrofoblasto crecidas, amorfas y multinucleadas. B: imagen de inmunohistoquímica para detección de gonadotropina coriónica humana, con marcaje del citoplasma de las células del sincitiotrofloblasto.

Worster, et al. ${ }^{2}$, quienes señalan que solo el $1 \%$ de todos los tumores de células germinales en los hombres corresponden a un coriocarcinoma. Acorde con lo reportado por Haas y Schmidt" ${ }^{11}$, con base en la clasificación británica de tumores testiculares el coriocarcinoma se considera un teratoma trofoblástico maligno por su estirpe histológica de células trofoblásticas, como mencionamos en la introducción. La presencia de células del sincitiotrofoblasto condiciona la elevación de la fracción $\beta$ de la gonadotropina coriónica humana y de la $\alpha$-fetoproteína. En nuestro paciente no se logró cuantificar los marcadores tumorales, ya que la presentación clínica fue abrupta y las manifestaciones clínicas fueron tardías y atípicas, aunque la elevación de la DHL a 2355 UI// coincide con lo reportado por Reilley y Pagliaro?.

No es de extrañar que la presentación clínica sea tardía, ya que la cultura de autoexploración genital en el sexo masculino es pobre, sobre todo en edades jóvenes, como en el caso de nuestro paciente de 19 años, que presentó aumento de volumen en el testículo derecho, pero que en el interrogatorio indirecto posterior a la cirugía la madre refirió desconocer el hallazgo. Pese a que de la segunda a la cuarta décadas de la vida es el rango de edad con más reporte de casos, tal como encontraron Fosado Gayosso, et al. ${ }^{12}$ en un joven de 25 años en quien se encontraron células trofoblásticas en varias estructuras digestivas, así como en el hígado, el cerebro y el pulmón, en el reporte de la autopsia, en nuestro paciente no hizo autopsia, pero en la figura $2 \mathrm{~B}$ se observa la presencia de lesiones nodulares hepáticas muy probablemente correspondientes a metástasis. Donohue, et al. ${ }^{13}$ explican que la gran variedad de líneas celulares inherentes a la formación de un tumor de células germinales es la condicionante de que los sitios de metástasis sean muy diversos para los tumores de células trofoblásticas.

La presentación agresiva, en conjunto con la detección tardía, de tumores gonadales en el hombre hacen que el pronóstico y la sobrevida sean desafortunados, e incluso que las manifestaciones extragonadales, como la hemorragia de tubo digestivo, sean el primer dato de alarma y que se sospechen muchas otras causas antes de imaginar que se trata de un coriocarcinoma metastásico. Martínez Ordaz, et al. ${ }^{14}$ explican que los coriocarcinomas son los tumores de células germinales con mayor formación neovascular, y que aun así la proliferación celular rápida condiciona una necrosis del propio tumor primario o metastásico, y eso explica la hemorragia como síntoma clínico en dos de sus tres casos reportados, que cabe señalar que se trataba de hombres de 24 y 19 años que, a semejanza de nuestro paciente de 19 años, presentaron hemorragia de tubo digestivo alto manifestada como evacuaciones melénicas como uno de sus síntomas más alarmantes. Es usual que el coriocarcinoma esté asociado a un adenocarcinoma desarrollado en otro sitio ajeno a las 
estructuras genitales ${ }^{15}$, bien descrito anteriormente por Nguyen ${ }^{10}$ en 1982, aunque esta forma de presentación es muy poco común, habiendo solo seis casos reportados de coriocarcinoma primario en el colon.

El conjunto de todos estos aspectos poco favorecedores para la vida de los pacientes hace que el diagnóstico sea muy tardío, e incluso en muchas ocasiones la causa documentada del sangrado de tubo digestivo en los pacientes sometidos a laparotomía se obtiene días e incluso meses más tarde por los reportes de los patólogos, quienes han colaborado formulando varias hipótesis para explicar la etiología del coriocarcinoma extragonadal: 1) que el tumor surge de la retención de células germinales que migraron anormalmente durante la embriogénesis; 2) que las metástasis ocurren de un tumor primario gonadal que revierte espontáneamente; y 3) que su desarrollo se produce como consecuencia de una diferenciación multidireccional de células madre en epitelio maligno, aunque recientemente se han reportado mutaciones en líneas germinales que se correlacionan con tumores extragonadales de células germinales, pero la aplicación de estas distinciones patológicas no se ha logrado exitosamente, según reportan Qiu, et al. ${ }^{16}$. En este caso de un joven de 19 años que evolucionó desfavorablemente y falleció en menos de 24 horas posquirúrgicas, la causa clara que condicionó la hemorragia de tubo digestivo logramos conocerla 1 mes después del procedimiento quirúrgico.

\section{Conclusiones}

El coriocarcinoma metastásico al tracto gastrointestinal es raro, lo que hace que la sospecha diagnóstica ante la presencia de un sangrado de tubo digestivo alto sea pobre. Incluso al encontrar un tumor en el intestino delgado y hacer resección, el diagnóstico puede ser tardío y ser el reporte de anatomía patológica el que otorgue la causa documentada del tumor que fue origen de la hemorragia digestiva.

\section{Agradecimientos}

Los autores agradecen al servicio de cirugía general y al servicio de anatomía patológica del Hospital Juárez de México por el apoyo brindado.

\section{Responsabilidades éticas}

Protección de personas y animales. Los autores declaran que para esta investigación no se han realizado experimentos en seres humanos ni en animales.

Confidencialidad de los datos. Los autores declaran que han seguido los protocolos de su centro de trabajo sobre la publicación de datos de pacientes.

Derecho a la privacidad y consentimiento informado. Los autores han obtenido el consentimiento informado de los pacientes y/o sujetos referidos en el artículo. Este documento obra en poder del autor de correspondencia.

\section{Financiamiento}

Este articulo no recibió financiamiento por ninguna entidad, del sector público ni privado.

\section{Conflicto de intereses}

No existe conflicto de intereses para la realización de este artículo.

\section{Bibliografía}

1. Jiang L, Wu I, Peng P. Primary choriocarcinoma of the colon: a case report and review of the literature. World J Surg Oncol. 2013;11:23.

2. Worster A, Sharma S, Mookadam F, Opie J. Acute presentation of choriocarcinoma: a case study and review of the literature. CJEM. 2002:4:111-4.

3. Yokoi K, Tanaka N, Furukawa K, Ishikawa N, Seya T, Horiba K, et al. Male choriocarcinoma with metastasis to the jejunum: a case report and review of the literature. J Nippon Med Sch. 2008;75:116-21.

4. Iyomasa S, Senda Y, Mizuno K, Miyake H, Amemiya T, Yamaguchi J, et al. Primary choriocarcinoma of the jejunum: report of a case. Surg Today. 2003:33:948-51.

5. Lowe K, Paterson J, Armstrong S, Walsh S, Groome M, Mowat C. Metastatic testicular choriocarcinoma: a rare cause of upper GI bleeding. ACG Case Rep J. 2015;3:36-8.

6. Shariat S, Duchene D, Kabbani W, Mucher Z, Lotan Y. Gastrointestinal hemorrhage as first manifestation of metastatic testicular tumor. Urology. 2005;66:1319.e21-4

7. Reilley M, Pagliaro L. Testicular choriocarcinoma: a rare variant that requires a unique treatment approach. Curr Oncol Rep. 2015;17:2.

8. Rejlekova K, Cursano M, De Giorgi U, Mego M. Severe complications in testicular germ cell tumors: the choriocarcinoma syndrome. Front Endocrinol (Lausanne). 2019;10:218

9. Molina Infante J, Beceiro Pedreño I, Ripoll Noiseux C, Marín Jiménez I, González Asanza C, Menchén Fernández-Pacheco P. Gastrointestinal hemorrhage due to metastatic choriocarcinoma with gastric and colonic involvement. Rev Esp Enferm Dig. 2004;96:77-80.

10. Nguyen G. Adenocarcinoma of the sigmoid colon with focal choriocarcinoma metaplasia. Dis Colon Rectum. 1982;25:230-4.

11. Haas R, Schmidt P. Testicular germ-cell tumors in childhood and adolescence. World J Urol. 1995;13:203-8.

12. Fosado Gayosso M, Pérez Hernández J, Bernal Sahagún F, Acevedo García C, Aguilar Ayala E, Pérez Espinosa J. Upper gastrointestinal bleeding as an initial manifestation of metastasis, secondary to a choriocarcinoma in a patient suffering from testicular mixed germ cell tumour. Rev Esp Enferm Dig. 2011;103:46-7.

13. Donohue J, Foster R, Little J, Loehrer P, Einhorn L. 1996. Biology of metastases and its clinical implications: testicular germ-cell tumors. World J Urol. 1996;14:197-203.

14. Martínez-Ordaz J, Gómez-Jiménez L, Ballinas-Oseguera G. Coriocarcinoma metastásico a yeyuno. Reporte de tres casos. Rev Gastroenterol Mex. 2012;77:143-7.

15. Le D, Austin R, Payne S, Dworkin M, Chappell M. Choriocarcinoma of the colon. Dis Colon Rectum. 2003;46:264-6.

16. Qiu J, Jia S, Li G. Incidence and prognosis factors of extragonadal choriocarcinoma in males: a population-based study. Cancer Manag Res. 2018;10:4565-73. 